\title{
Single-expansion EBCM computations for osculating spheres
}

\author{
Michael I. Mishchenko ${ }^{\mathrm{a}, *}$ and Gorden Videen \\ ${ }^{a}$ NASA Goddard Institute for Space Studies, 2880 Broadway, New York, NY 10025, U.S.A \\ ${ }^{\mathrm{b}}$ Army Research Laboratory AMSRL-IS-EE, 2800 Powder Mill Road, Adelphi, \\ MD 20783-1197, U.S.A.
}

\begin{abstract}
We show that the standard, single-expansion extended boundary condition method provides convergent scattering results for osculating dielectric spheres and discuss the implications of this result.
\end{abstract}

*Corresponding author. Fax: (001) 212678 5622; e-mail: crmim@giss.nasa.gov. 


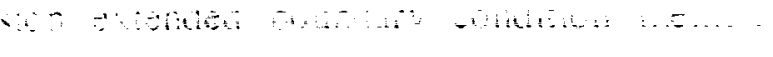

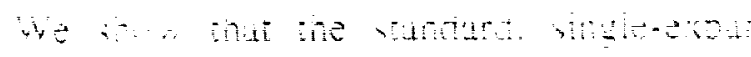




\section{Introduction}

The extended boundary condition method (EBCM), otherwise known as the single-expansion version of the $T$-matrix method, is an exact technique for computing electromagnetic scattering by small particles based on numerically solving Maxwell's equations [1, 2]. Although in principle this technique can be applied to arbitrarily shaped particles, most practical applications of EBCM have dealt with rotationally symmetric particles such as spheroids, finite circular cylinders, and so-called Chebyshev particles [2]. In this Brief Communication we apply EBCM to a new class of particles, namely, osculating spheres. This research is motivated not only by the natural desire to expand the range of shapes treated with EBCM, but also by the fact that the application of the multi-expansion superposition $T$-matrix method (STM) to osculating spheres seems to produce divergent results $[3,4]$.

\section{Computations}

Unlike STM [5], EBCM uses a single expansion of the incident, internal, and scattered fields in vector spherical wave functions. Computations for osculating spheres (i.e., spheres with the distance between their centers smaller than the sum of their radii; see Fig. 1) required a rather straightforward modification of the single-expansion $T$-matrix code described in [6] and available at http://www.giss.nasa.gov/ crmim. Specifically, we have added a simple subroutine which computes the shape of the particle, $r(\vartheta, \varphi)$, and the partial derivative $\partial r(\vartheta, \varphi) / \partial \vartheta$. The shape of a particle formed by a pair of identical osculating spheres in the spherical coordinate system with the $z$ axis directed along the line connecting the centers of the spheres (Fig. 1) is given by the following simple expression:

$$
r(\vartheta, \varphi)= \begin{cases}R\left(f \cos \vartheta+\sqrt{1-f^{2} \sin ^{2} \vartheta}\right), & \vartheta<\pi / 2, \\ R\left(-f \cos \vartheta+\sqrt{1-f^{2} \sin ^{2} \vartheta}\right), & \vartheta>\pi / 2,\end{cases}
$$

where $R$ is the radius of the component spheres, $f=d /(2 R)$, and $d$ is the distance between the sphere centers. Furthermore, 


$$
\frac{\partial r(\vartheta, \varphi)}{\partial \vartheta}= \begin{cases}-R f \sin \vartheta\left[1+\frac{f \cos \vartheta}{\sqrt{1-f^{2} \sin ^{2} \vartheta}}\right], & \vartheta<\pi / 2, \\ R f \sin \vartheta\left[1-\frac{f \cos \vartheta}{\sqrt{1-f^{2} \sin ^{2} \vartheta}}\right], & \vartheta>\pi / 2 .\end{cases}
$$

The $T$-matrix code computes the optical cross sections, the asymmetry parameter of the phase function, and the elements of the scattering matrix for randomly oriented particles. Since osculating spheres are particles with a plane of symmetry, the scattering matrix has a simple block-diagonal structure,

$$
\mathbf{F}(\Theta)=\left[\begin{array}{cccc}
a_{1}(\Theta) & b_{1}(\Theta) & 0 & 0 \\
b_{1}(\Theta) & a_{2}(\Theta) & 0 & 0 \\
0 & 0 & a_{3}(\Theta) & b_{2}(\Theta) \\
0 & 0 & -b_{2}(\Theta) & a_{4}(\Theta)
\end{array}\right],
$$

where $\Theta$ is the scattering angle, and has only six independent elements [7]. The (1,1)-element of the scattering matrix (i.e., the phase function) satisfies the standard normalization condition,

$$
\frac{1}{2} \int_{0}^{\pi} a_{1}(\Theta) \sin \Theta \mathrm{d} \Theta=1
$$

We have found that although the accuracy of single-expansion $T$-matrix computations for osculating spheres depends on the particle refractive index $m$ and size parameter $x=2 \pi R / \lambda$ ( $\lambda$ is wavelength) as well as on $f$, convergent results can be obtained for a rather wide range of these parameters. As an example, Fig. 1 and Table 1 show results computed with an extendedprecision FORTRAN code for $m=1.31, R=0.5 \mu \mathrm{m}, \lambda=0.6283 \mu \mathrm{m}$, and $f=0$ (single sphere), 0.2 , and 0.5 . For comparison, we also show results for a bisphere with touching components $f=$ 1) obtained with the superposition $T$-matrix code described in [8]. In all cases the size of the $T$ matrix was increased in unit steps until the extinction and scattering cross sections converged within $0.01 \%$. The physical correctness of the results was checked using the general relationships derived in $[9,10]$. Furthermore, we have made sure that $\mathrm{EBCM}$ results for $f=0$ (single sphere) exactly reproduce the corresponding Lorenz-Mie results.

Not surprisingly, the single-sphere curves in Fig. 1 exhibit the largest amplitude of oscillations caused by interference effects, whereas the amplitude of oscillations for other 
particles is reduced by averaging over orientations. The growth of the average projected area with increasing $f$ causes a notable increase of the extinction cross section (Table 1) and the forward-scattering phase function value, $a_{1}\left(0^{\circ}\right)$. Although for spheres $a_{2}(\Theta) \equiv a_{1}(\Theta)$ and $a_{4}(\Theta) \equiv a_{3}(\Theta)$, the results for nonspherical particles with $f>0$ in Fig. 1 show significant differences between these scattering matrix elements.

Note that even with the extended-precision code, we could not obtain convergence for $f>$ 0.5 for the same $m, R$, and $\lambda$. Apparently, this can be explained by the sensitivity of singleexpansion EBCM computations to increasing concavity of the particle shape noted earlier by Mugnai and Wiscombe [11] in their calculations for Chebyshev particles.

\section{Discussion and Conclusions}

Our results demonstrate that EBCM can be successfully applied to osculating spheres. On the other hand, the results of $[3,4]$ suggest that the multi-expansion STM, while providing convergent results for bispheres with separated or touching components $(f \geq 1)$ [8], produces divergent results for osculating spheres $(f<1)$. Furthermore, the performance of EBCM improves with decreasing $f$, in contrast to the behavior of STM. It is important to realize that STM for bispheres with touching or separated components does not rely on the so-called Rayleigh hypothesis (RH), but becomes dependent on $\mathrm{RH}$ for $f<1$. (RH states that the expansion of the scattered field in outgoing spherical functions is valid in the region between the particle surface and its smallest circumscribing sphere.) Similar convergence problems are encountered with the point-matching technique, which also explicitly relies on RH [12]. On the other hand, the derivation of EBCM in [13] completely avoids the use of RH. Therefore, it seems logical to suggest that the success of EBCM in computations for osculating spheres may be explained by EBCM not relying on $\mathrm{RH}$.

This result may be important since it has been shown that EBCM can be derived from RH [13]. The title and conclusions of [14] may seem to suggest that EBCM is equivalent to the method of RH. However, the fact that EBCM can be derived from RH only means that RH is a sufficient condition of validity of EBCM, but not the necessary condition. Therefore, one should not exclude the possibility that EBCM may be valid even when RH is not. 


\section{Acknowledgements}

We thank David Brown and Cornelis V. M. van der Mee for careful reading of the manuscript and valuable comments. This research was sponsored by the NASA Radiation Science Program managed by Robert Curran.

\section{References}

1. Waterman P. C., Symmetry, unitarity, and geometry in electromagnetic scattering, Phys. Rev. D, 1971, 3, 825-839.

2. Mishchenko, M. I., Travis, L. D. and Macke, A., T-matrix method and its applications. In Light Scattering by Nonspherical Particles: Theory, Measurements, and Applications, M. I. Mishchenko, J. W. Hovenier and L. D. Travis, eds., Academic Press, San Diego, 1999.

3. Videen, G., Ngo, D. and Hart, M. B., Light scattering by a pair of conducting, osculating spheres, Opt. Commun., 1996, 125, 275-287.

4. Ngo, D., Videen, G. and Dalling, R., Chaotic light scattering from a system of osculating, conducting spheres, Phys. Lett. A, 1997, 227, 197-202.

5. Fuller, K. A. and Mackowski, D. W., Electromagnetic scattering by compounded spherical particles. In Light Scattering by Nonspherical Particles: Theory, Measurements, and Applications, M. I. Mishchenko, J. W. Hovenier and L. D. Travis, eds., Academic Press, San Diego, 1999.

6. Mishchenko, M. I. and Travis, L. D., Capabilities and limitations of a current FORTRAN implementation of the T-matrix method for randomly oriented, rotationally symmetric scatterers, J. Quant. Spectrosc. Radiat. Transfer, 1998, 60, 309-324.

7. van de Hulst, H. C., Light Scattering by Small Particles, Wiley, New York, 1957 (also Dover, New York, 1981).

8. Mishchenko, M. I. and Mackowski, D. W., Light scattering by randomly oriented bispheres, Opt. Lett., 1994, 19, $1604-1606$.

9. van der Mee, C. V. M. and Hovenier, J. W., Expansion coefficients in polarized light transfer, Astron. Astrophys., 1990, 228, 559-568. 
10. Hovenier, J. W. and van der Mee, C. V. M., Basic relationships for matrices describing scattering by small particles. In Light Scattering by Nonspherical Particles: Theory, Measurements, and Applications, M. I. Mishchenko, J. W. Hovenier and L. D. Travis, eds., Academic Press, San Diego, 1999.

11. Mugnai, A. and Wiscombe, W. J., Scattering from nonspherical Chebyshev particles. 1: Cross sections, single-scattering albedo, asymmetry factor, and backscattered fraction, Appl. Opt., $1986,25,1235-1244$.

12. Oguchi, T., Scattering from hydrometeors: A survey, Radio Sci., 1981, 16, 691-730.

13. Waterman, P. C., Matrix methods in potential theory and electromagnetic scattering, J. Appl. Phys., 1979, 50, 4550-4566.

14. Schmidt, K., Rother, T. and Wauer, J., The equivalence of the extended boundary condition and the continuity conditions for solving electromagnetic scattering problems, Opt. Commun., 1998, 150, 1-4. 


\section{FIGURE CAPTIONS}

Fig. 1. Spherical coordinates used to specify the shape of an osculating sphere with equal components.

Fig. 2. Elements of the scattering matrix versus scattering angle for randomly oriented osculating spheres with $f=1$ (bisphere with touching components), $0.5,0.2$, and 0 (single sphere).

Table 1. Extinction cross section and asymmetry parameter of the phase function for randomly oriented osculating spheres with $f=1$ (bisphere with touching components), $0.5,0.2$, and 0 (single sphere)

\begin{tabular}{ccc}
\hline$f$ & $C_{\text {ext }}\left(\mu \mathrm{m}^{2}\right)$ & $g$ \\
\hline & & \\
1 & 4.861 & 0.8573 \\
0.5 & 4.185 & 0.8617 \\
0.2 & 3.360 & 0.8615 \\
0 & 2.654 & 0.8550 \\
\hline
\end{tabular}




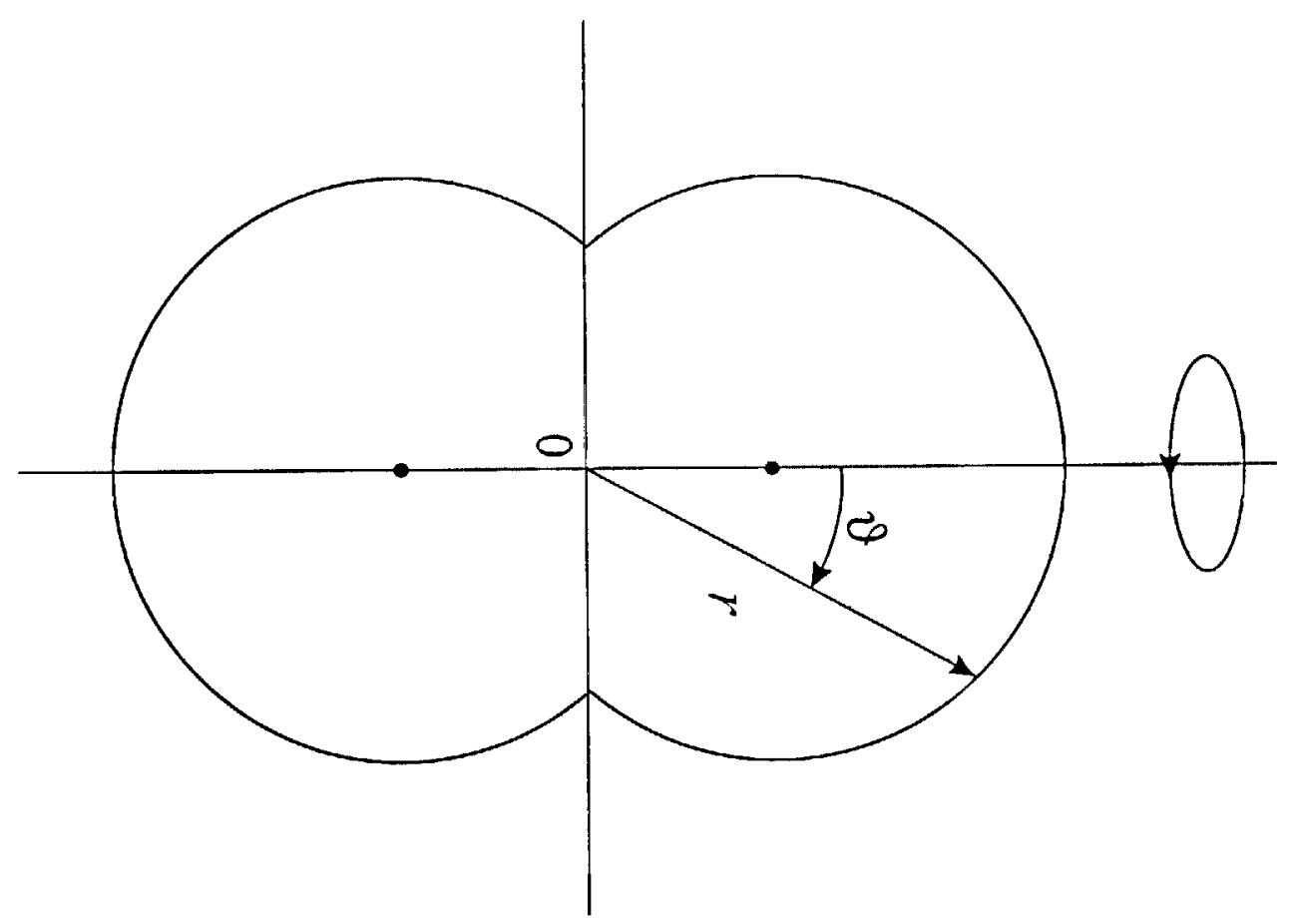



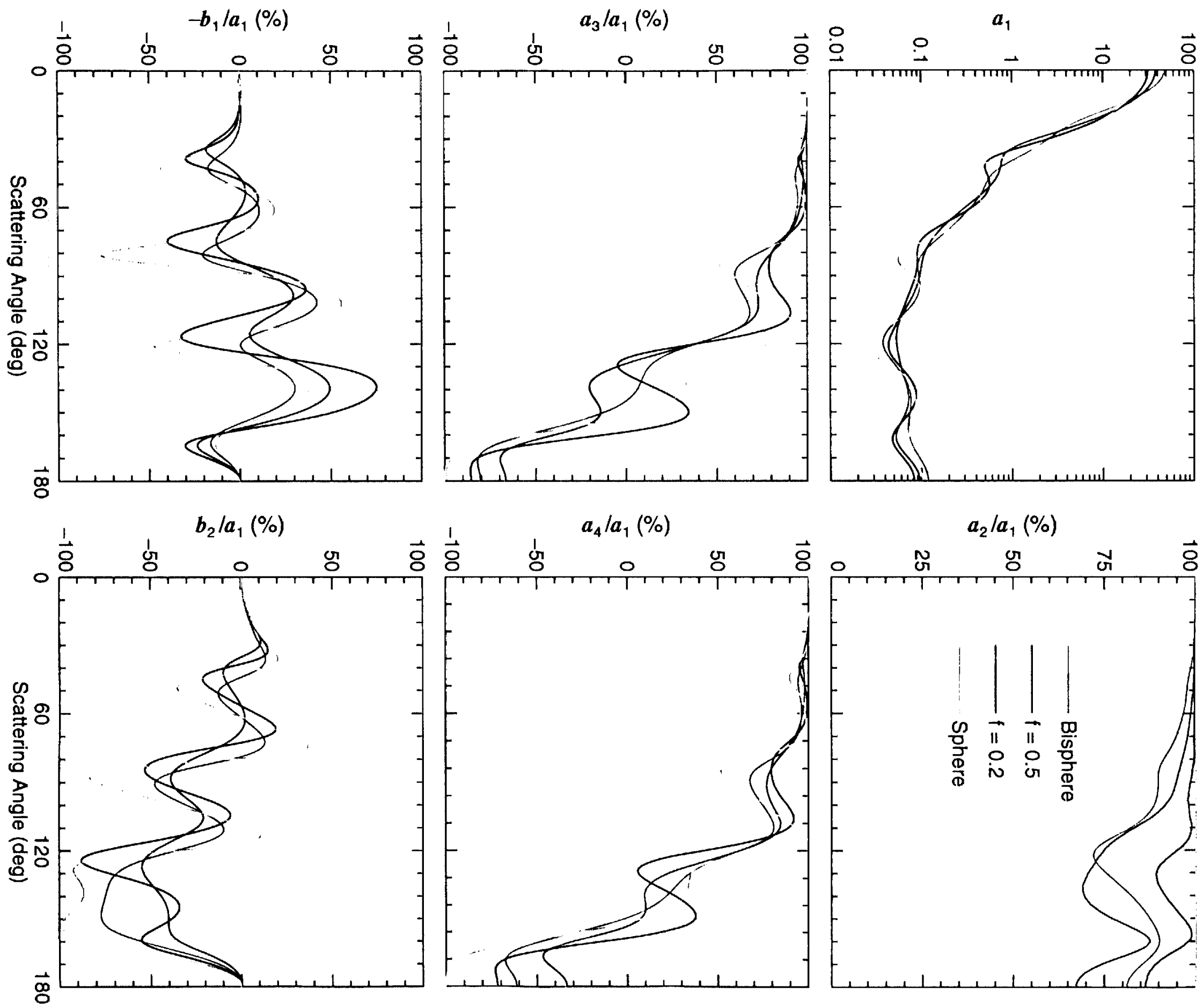